\title{
Allocation of Fetal Heart Tones During Non-stop Monitoring of Pregnant Women
}

\author{
Kiseleva E.Yu., Zhdanov D.S., \\ Zemlyakov I.Yu., Vaganova E.V. \\ Tomsk, Diagnostika+ LLC \\ E-mail: info@diagnostic.tom.ru
}

\author{
Yuryev S.Yu. \\ Tomsk, Siberian State Medical University \\ E-mail: sergeiyuriev@gmail.com
}

\begin{abstract}
Annotation - The article is to study the system of non-stop daily monitoring of the state of the maternal and the fetal circulatory systems using acoustic sensors. After preliminary preparation of the phonocardiogram heart tones of the fetus and the pregnant woman were extracted using Shannon energy. The obtained data was separated, followed by calculation of the heart beats. As a result, software algorithms for calculating heart beats at a particular time (30 seconds to 6 hours) have been developed and implemented. The measurement error ranges from 1 to 6 beats per minute. The accuracy of the software and algorithmic support was estimated by comparing the results obtained during parallel cardiotocography, taken as the reference method.
\end{abstract}

Keywords: cardiotocography, phonocardiography, allocation of heart tones separation, daily monitoring

\section{INTRODUCTION}

The problem of monitoring the state of the circulatory systems of the fetus and the pregnant woman was repeatedly raised in the world scientific literature.

There is an existing method of non-invasive assessment of fetal well-being during the second and third trimester of pregnancy on the basis of the analysis of heart rate (cardiotocography, CTG).

The present method is based on the use of ultrasound (U/S) investigation under outpatient treatment. However according to the Federal standards, approved by the Health Ministry of the Russian Federation (order № 662, Sept.14, 2006) women with normal pregnancy in general are assigned to two sessions of the CTG.

This is due to the fact that it is still not clearly proved whether long-term influence of ultrasound energy is safe for the fetus.

Thus, the practical health care lacks a safe, objective and inexpensive method for dynamic monitoring of the fetus with an optimal ratio of sensitivity and specificity, which can be used from the $23 \mathrm{~d}$ week of pregnancy and does not require a hospital stay of the woman.

Currently fetal electrocardiogram (ECG) using electrodes imposed on woman's abdominal wall [4] is considered a secure method of daily monitoring.

At present Monica AN24, MONICA Health Care, UK is only portable medical device intended for fetal heart rate assessment in the world. The functional principle is based on measuring of the maternal and the fetal heart rates.

The device is a hardware system of autonomous portable battery-powered recorder (about 350 gr.) and computer workstation of the doctor with specialized software.

The ECG signals of the fetus are registered by 5 cardio electrodes, located on the anterior abdominal wall of the pregnant woman. They are recorded in the data flash memory. If necessary, the data are transferred to the computer workstation of the doctor via Bluetooth or USB. Battery charge provides autonomous operation of the recorder for 20-22 hours.

Receiving of high quality cardiogram and assessing heart rate of the fetus using MONICA AN24 can only be possible with limited mobility of the patient (even better in the supine position).

According to the published data about daily monitoring of the 72 women at home, only 64 records (approximately 89\%) can be considered successful [6].

As operating experience shows the dynamic pregnancy monitoring using MONICA AN24 almost excludes antenatal fetal death. It determines the degree of individual risk of prenatal complications in spontaneous labor, and reduces the frequency of births of children with severe injuries of central nervous system (CNS) caused by hypoxia. The use of the device also releases doctors from false positive decisions.

In Russia, the cost of one kit Monica AN24, including software for workplace of an obstetrician and 4 portable recorders is about $€ 12,500$. By the end of 2015 at least 21 sets were sold in Russia.

There are some developments of similar systems in Tomsk (joint project of Tomsk Polytechnic University and Siberian State Medical University, Russia), and in Kharkov (Scientific-technologic Center of radioelectronic medical devices and technologies XAI-Medica, Ukraine).

Despite the advantages of ECG (accurate measurement of R-R interval, easy and comfortable to use), allocation of heart tones, requires strong computing resources. This is due to the high sensitivity of the method, which makes it difficult to separate the fetal heart tones against electric miotic activity of skeletal muscles of the mother.

The analysis of the maternal ECG requires a large amount of computing resources. It complicates the real time assessment of the fetal heart rate [4].

Therefore, practical healthcare needs a safe, objective and inexpensive method for dynamic 
monitoring of the fetus with an optimal ratio of sensitivity and specificity, which can be used from the $23 \mathrm{~d}$ week of pregnancy and does not require a hospital stay.

Thus the design team of $R \& D$ company Diagnostika+ LLC proposed the development of a portable fetal monitor based on acoustic recording of fetal heart tones (phonocardiograms) as they are not aware of the devices similar in operability available.

\section{MATERIALS AND METHODS}

The basic principle of the method developed by the team is allocation of heart tones of the fetus and the mother against the background noise or interference.

The developed method is passive, has no negative effects on the human body and can be used for twenty-four-hour heart tones recording against uncontrollable noises (breathing, talking, work of equipment, clothes rubbing on the acoustic sensor, etc.).

For verification of the method a medicotechnical experiment for obtaining phonocardiograms was conducted in an obstetrical clinic . All requirements for personal data security of the patients were followed.

Data were collected in Siberian Federal Research and Clinical Centre, Seversk) and Prenatal Health Centre, Tomsk).

28 patients aged 19 to 39 with a normal pregnancy took part in the experiment. The average duration of the recording was 31 minutes. In order to control the quality of allocation of the maternal and the fetal heart tones the registration was carried along with the approved ultrasound procedure (CTG).

Fetal heart tones were detected and registered by polymorphic piezoceramic sensors connected to the battery-operated model recorder.

Signal sampling frequency was determined by its characteristics and experimental conditions. The digitized signal was recorded on the data flash memory (Micro-SD) in the format of audio Windows WAVEfile.

A computer workstation of the doctor includes a set of functions providing preliminary processing and analysis of acoustic signals of the the maternal and the fetal circulatory systems. Pre-processing functions include digital filters (finite impulse response), ranging from 2 to 51.The order of the implemented digital filters depends on the noise level of the analyzed signal.Signal processing results are presented in the form of text files and on-screen forms containing charts, tables and graphs.

The final preparation of signals and allocation of heart sounds were carried out on a workstation with a prototype of a computer workstation of the doctor.

The results of the experiment showed that the duration of a tone of 1 heart beat of a mother is about $400 \mathrm{~ms}$, and the one of a fetus is about $300 \mathrm{~ms}$.

The frequency spectrum of the both is similar, and the frequency range of acoustic vibrations in both cases reaches 0 to $70 \mathrm{~Hz}$. According to the reference ultrasonic CTG in all experiments heart rate of a fetus ranged from 126 to $160 \mathrm{~min}^{-1}$, heart rate of a mother ranged from 76 to $88 \mathrm{~min}^{-1}$.

For the allocation of heart tones Shannon energy has been used together with additional criteria for evaluating the resulting energy profile. The choice of method is related to its high efficiency in the allocation and identification of tones of heart against the background noise and interference [1].

In addition, unlike wavelet analysis the method does not require separating of the test signal band into sub-bands [3,5]. The following tasked were performed during application of the method:

The range of operating frequency and method of digital filtering to reduce noise and interference, suitable for the correct signal analysis has been chosen. - The algorithms for Shannon energy analysis for identification of heart sounds have been developed.

- $\quad$ The artifacts of Shannon energy have been removed and the periods of heart sounds have been allocated.

During the experimental data processing, it was found that the best option to suppress interference and noise in the already filtered signal is additional filtering by the 4th order of Daubechies wavelet. As a result of such filtering the output signal does not contain noise components and coarse clicks. Thus, the further processing, as unlike the windowed Fourier transformation [2], was performed correctly. The wavelet filter results are shown in Figure 1.

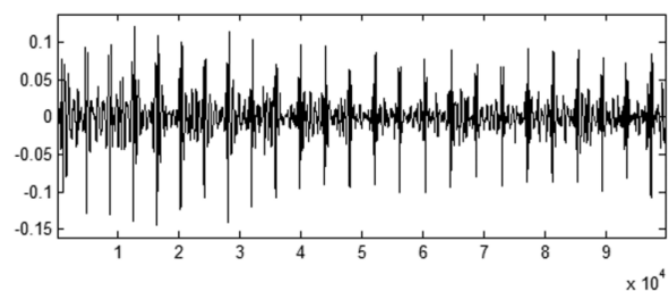

A.

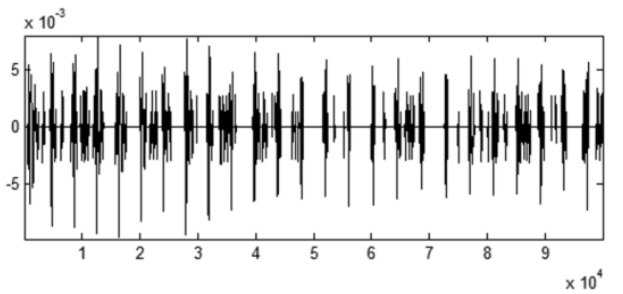

B.

Figure 1. Wavelet-filtering of a fetal

phonocardiogram. A. Original signal. B. Filtering result

The next task was to use the method of determination of Shannon energy level in a phonocardiogram in order to allocate and identify heart sounds.

Two criteria were used: the duration of power output peak which should not be less than $70 \%$ of the duration of a heart tone (300-400 ms), and its amplitude.

For identification of heart tones the level Shannon free energy was set within $20-25 \%$ of its maximum emission peak amplitude. All free energy 
fluctuations below this level were identified as noise (Figure 2).

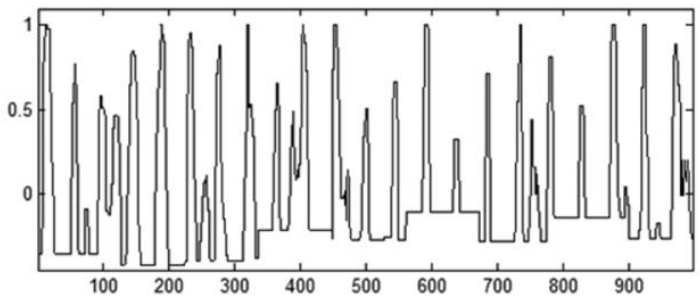

A.

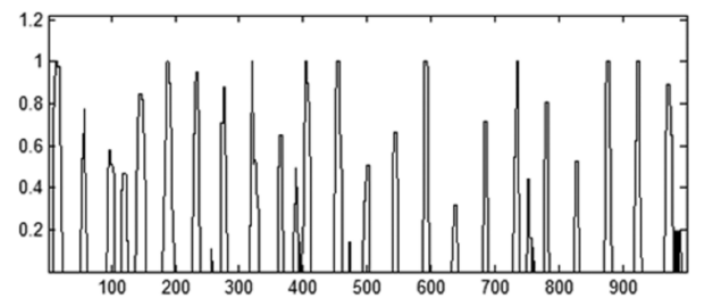

B.

Figure 2. Shannon energy profile applied for a fetal phonocardiogram (Fig. 1). A. Original value Б.

Normalized value

Construction of the Shannon energy profile is followed by identification of separated heart tones. This procedure was complicated by artifacts in the energy profile: splitting of the peaks and the appearance of phantom signals. Splitting of the peak occurs due to the detection of short energy emissions not related to one tone of the heart rhythm. It is due to a non-monotonic dependence of Shannon energy on time.

Phantom signals occurring from the unfiltered noise, appeared as short additional peaks, falling out of a consecutive series of events with a similar period.

The criteria for combining several short peaks in one was that the time intervals between them within 1 single tone (less than $300 \mathrm{~ms}$ ) were significantly smaller than the ones between two adjacent heart tones (350-400 ms).

Single short Shannon free energy emissions lasting less than $100 \mathrm{~ms}$ were qualified as noise and were not taken into account. The result of the applied approach is shown in Figure 3:

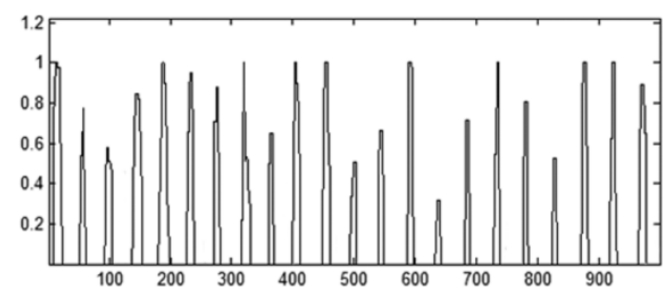

Figure3. Removal of artifacts in the Shannon energy profile of a phonocardiogram and allocation of heart tones (Fig. 2)

During the experiment the precision of the analytical algorithms and recognition of heart tones of the fetus and the mother reached 3 to 6 beats per minute compared to the reference method of ultrasound CTG.

From the point of view of the existing statistical approach during heart rates constructing in diagram form this error is insignificant. Multiple processing of the same phonocardiogram using the proposed methods also gave similar results. That indicates the stability of the developed algorithms.

The signal processing of mother's heart rate was similar to signal processing of fetal heart rate. These aspects have not been studied in the present paper. The comparison of data (maternal and the fetal heart rates) received by ultrasonic and phono cardiographic measurement principles is shown in Table 1:

Table 1.

The comparison of ultrasonic and phono cardiographic measurement principles

\begin{tabular}{|l|l|l|}
\hline \multicolumn{1}{|c|}{ Parameter } & $\begin{array}{l}\text { Ultrasonic } \\
\text { principle }\end{array}$ & $\begin{array}{l}\text { Phono } \\
\text { cardiographic } \\
\text { principle }\end{array}$ \\
\hline Fetal heart rate, $\mathrm{min}^{-1}$ & $126 \ldots 160$ & $120 \ldots 165$ \\
\hline $\begin{array}{l}\text { Maternal heart rate, } \\
\text { min }^{-1}\end{array}$ & $76 \ldots 88$ & $75 \ldots 88$ \\
\hline
\end{tabular}

The results received (the maternal and the fetal heart rates), were compared with the referential data obtained during the CTG procedure.

The absolute value of fetal CTG measurement methods based on the phonocardiographic principle differed from fetal CTG based on the ultrasonic principle for $3 \ldots 6$ beats / min, resulting in an error of less than $3 \%$. According to the team of the developers, such error of the phonocardiographic assessment method, can be considered insignificant.

The processing time on a workstation with 2core Intel Pentium i5 processor with 8GB RAM, depended mainly on the duration of the original record. The processing of records of different duration is presented in Table 2.

Table 2.

Signal processing time of the fetal heart rate or the maternal heart rate

\begin{tabular}{|l|l|}
\hline Signal duration, minutes & Processing time, seconds \\
\hline 18 & $25-30$ \\
\hline $100-120$ & $90-120$ \\
\hline 8 & $250-300$ \\
\hline
\end{tabular}

\section{RESULTS AND DISCUSSION}

Therefore, the proposed procedure of preliminary preparation of the phonocardiograms, as well as the methods of analysis for heart rate determining allowed to some extent to solve the problem of noise-resistant method for twenty-four-hour non-stop monitoring of the parameters of the maternal 
and the fetal circulatory systems. At present, the developed algorithms are implemented in the form of a complex prototype of server applications for non-stop monitoring of the maternal and the fetal circulatory systems.

Suggestions for further research include individual portable devices, based on the developed algorithms. Computing power of modern microcontrollers with low power consumption is enough to solve the above listed problems in real-time.

\section{ACKNOWLEDGMENT}

The work was performed within the framework of the Agreement № 14.579.21.0019 (unique agreement identifier RFMEFI57914X0019) on the «Development of the device for daily monitoring of the fetus and the mother during pregnancy by controlling the parameters of the circulatory system based on the acoustic data», signed between the R\&D company Diagnostika+ LLC and the Ministry of Education and Science of the Russian Federation.

\section{REFERENCES}

[1] Abbas A. K., Bassam R. Phonocardiography Signal Processing. Synthesis Lectures on Biomedical Engineering. - Morgan and Claypool publisher. 2009. $-194 \mathrm{pp}$.

[2] Balasubramaniam B. Efficient Computation of Phonocardiographic Signal Analysis in Digital Signal Processor Based System / B. Balasubramaniam, B. Nedumaran // International Journal of Computer Theory and Engineering. - 2010. - V. 2, № 4. - P. 660-664.

[3] Rangayyan R.M., Lehner R.J Phonocardiogram signal analysis: a review// CRC Critical Review un Biomedical Engineering. 1998. V. 15. No.3. P. 211-223.

[4] Rangayyan R.M. Biomedical Signal Analysis. - IEEE Press/Wiley, N.Y., 2002. - 514 pp.

[5] Sava H., Pibarot P., Durand L. Application of matching pursuit method for structural decomposition and averaging of phonocardiographic signals. In Medical and Biological Engineering and Computing. 1998. V.36. No.3 P.302-308

[6] Zubair R., Zarko A. Continuous remote fetal monitoring with MONICA AN24 during home induction of labor. / Am. J. Obstet. Gynecol. Vol. 204, Iss. 1, Suppl. Jan. 2011. - P. S263. 